\title{
Volt-VAr Control and Energy Storage Device Operation to Improve the Electric Vehicle Charging Coordination in Unbalanced Distribution Networks
}

\author{
Carlos Sabillon-Antunez, Ozy D. Melgar-Dominguez, John F. Franco, Member, IEEE, \\ Marina Lavorato, Member, IEEE, and Marcos J. Rider, Senior Member, IEEE
}

\begin{abstract}
In this paper, a new approach is presented to solve the electric vehicle charging coordination (EVCC) problem considering Volt-VAr control, energy storage device (ESD) operation and dispatchable distributed generation (DG) available in threephase unbalanced electrical distribution networks (EDNs). Dynamic scheduling for the EVCC is proposed through a step-by-step methodology, which solves a mixed integer linear programming (MILP) problem for the whole time period. The objective is to minimize the total cost of energy purchased from the substation and DG units, the cost of energy curtailment on electric vehicles, the cost of energy injected from the ESDs, and the cost of energy curtailment on the ESDs. The Volt-VAr control considers the management of on-load tap changers, voltage regulators, and switchable capacitors installed along the grid. Furthermore, the formulation takes into account the voltage dependence of the loads, while the steady-state operation of the unbalanced distribution systems is modeled using linear constraints. The proposed model was tested in a 178-node three-phase unbalanced EDN considering a one-day time period.
\end{abstract}

Index Terms-Electric vehicle charging coordination (EVCC) problem, energy storage devices (ESDs), mixed integer linear programming (MILP), Volt-VAr control, voltage-dependent load model.

\section{ACRONYMS}

CB Capacitor bank

DG Distributed generation

DoD Depth of discharge

EDN Electrical distribution network

ESD Energy storage device

Manuscript received October 19, 2016; revised February 10, 2017; accepted April 11, 2017. Date of publication April 18, 2017; date of current version September 15, 2017. This work was supported by the Brazilian institutions CAPES, CNPq, and FAPESP. Paper no. TSTE-00805-2016. (Corresponding author: Marcos J. Rider.)

C. Sabillon and O. D. Melgar-Dominguez are with the Department of Electrical Engineering, UNESP - São Paulo State University, Ilha Solteira 15385-000, São Paulo, Brazil (e-mail: cfsa27@gmail.com; ozzy. damedo@gmail.com).

J. F. Franco is with the UNESP - São Paulo State University, Rosana 19273000, São Paulo, Brazil (e-mail: j.f.franco@ieee.org).

M. Lavorato is with the Electrical Engineering Faculty, PUC/CAMPINAS Pontifical Catholic University of Campinas, Campinas 13086-900, São Paulo, Brazil (e-mail: marina.oliveira@puc-campinas.edu.br).

M. J. Rider is with the Department of Systems and Energy, University of Campinas, Campinas 13083-852, São Paulo, Brazil (e-mail: mjrider@dsee. fee.unicamp.br).

Color versions of one or more of the figures in this paper are available online at http://ieeexplore.ieee.org.

Digital Object Identifier 10.1109/TSTE.2017.2695195

$\begin{array}{ll}\text { EV } & \text { Electric vehicle } \\ \text { EVCC } & \text { Electric vehicle charging coordination } \\ \text { MILP } & \text { Mixed integer linear programming } \\ \text { MINLP } & \text { Mixed integer nonlinear programming } \\ \text { OLTC } & \text { On-load tap changer } \\ \text { SOC } & \text { Initial state of charge } \\ \text { VR } & \text { Voltage regulator } \\ \text { VVC } & \text { Volt VAr control }\end{array}$

NOMENCLATURE

Constants:

$\zeta_{n, t}^{G} \quad$ Energy cost at node $n$ at time $t$

$\zeta_{c}^{E V}, \zeta_{c}^{S D} \quad$ EV and ESD energy curtailment costs

$\zeta^{S D} \quad$ Unit cost for the active power taken from the

$\alpha_{n, f}, \beta_{n, f}$ ESD

Load voltage dependence parameters of the active and reactive power at node $n$, phase $f$

$\gamma(e, m, f) \quad$ Function that indicates whether EV $e$ is connected at node $m$ and phase $f$

$\gamma(u, m, f)$

Function that indicates whether ESD $u$ is connected at node $m$ and phase $f$

$\eta_{e}$

$\eta_{u}^{+}, \eta_{u}^{-}$

$\Delta_{t}$ $\Delta^{c b}, \Delta^{v r}$

Charging efficiency of the charging station for $\mathrm{EV} e$

Charging and discharging efficiencies of the charging station for ESD $u$

Duration of time interval $t$

Maximum number of operations allowable over the time period for the CBs and VRs

$\phi_{n} \quad$ Minimum power factor for the operation of the DG at node $n$

$\bar{B}_{n} \quad$ Maximum number of CB modules at node $n$

$B_{m n, f}^{s h}$

$\bar{E}_{e}^{E V}, \bar{E}_{u}^{S D}$

$E_{e}^{i n i}$

$E_{u}^{S D_{f}}$

Shunt susceptance of circuit $m n$, phase $f$

Energy capacity of EV $e$ and ESD $u$

Initial state of charge of EV $e$

Goal for the state of charge of ESD $u$ at the end of the time period

Initial state of charge of $\operatorname{ESD} u$

$E_{u}^{S D i}$
$\bar{I}_{m n}$
$I_{m n, f, t}^{i m^{*}}, I_{m n, f, t}^{r e^{*}}$

Maximum current flow magnitude of circuit $m n$

Imaginary and real part of the estimated current of the VR in circuit $m n$, phase $f$, time $t$ 


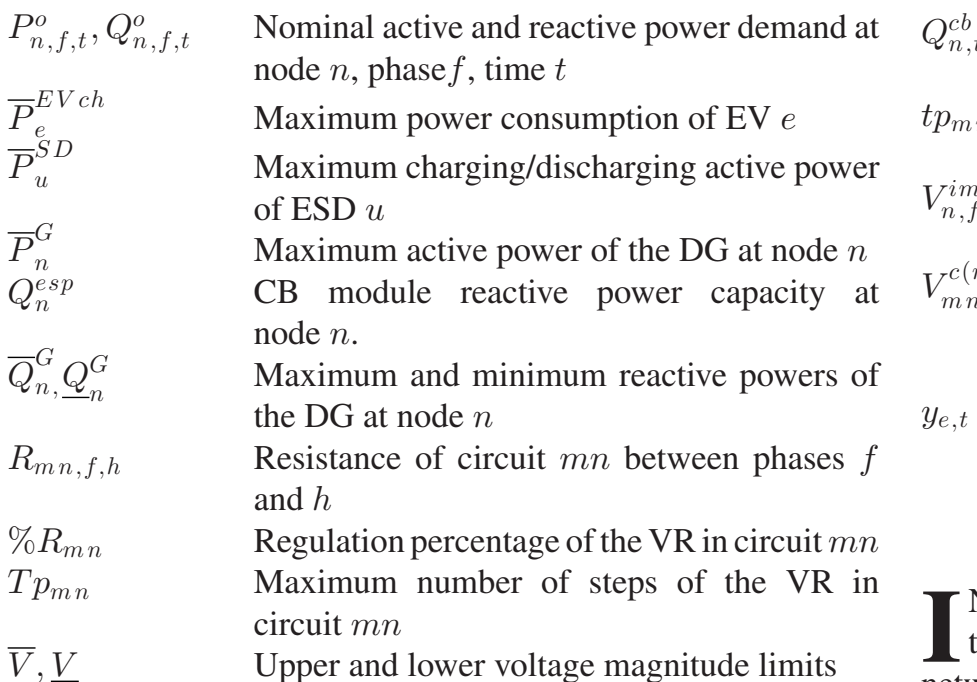

$V_{o} \quad$ Nominal voltage

$V_{n, f, t}^{i m^{*}}, V_{n, f, t}^{r e^{*}} \quad$ Imaginary and real part of the estimated voltage at node $n$, phase $f$, time $t$

$X_{m n, f, h}$

Variables:

$B_{n, t}$

$b t_{m n, f, t, k}$

$E_{e}^{E V}$

$E_{e}^{E V c}$

$E_{u, T}^{S D}$

$E_{u}^{S D C}$

$I_{m n, f, t, k}^{c(r e)}$

$I_{n, t}^{c b i m}, I_{n, t}^{c b r e}$

$I_{n, f, t}^{D i m}, I_{n, f, t}^{D r e}$

$I_{e, t}^{E V i m}, I_{e, t}^{E V r e}$

$I_{n, f, t}^{G i m}, I_{n, f, t}^{G r e}$

$I_{m n, f, t}^{i m}, I_{m n, f, t}^{r e}$

$I_{u, t}^{S D i m}, I_{u, t}^{S D r e}$

$P_{n, f, t}^{D}, Q_{n, f, t}^{D}$

$P_{n, t}^{G}, Q_{n, t}^{G}$

$P_{e, t}^{E V}$

$P_{u, t}^{S D^{+}}, P_{u, t}^{S D^{-}}$
Modules of the CB in operation at node $n$, time $t$

Binary variable that describes the tap position of the VR in circuit $m n$, phase $f$, time $t$, position $k$

Energy of EV $e$ at the end of the time period

Energy curtailment on EV $e$ at the end of the time period

Energy of ESD $u$ at the end of the time period

Energy curtailment on $\operatorname{ESD} u$ at the end of the time period

Real part of the VR current calculation auxiliary variable in circuit $m n$, phase $f$, time $t$, position $k$

Imaginary and real part of the current generated by the $\mathrm{CB}$ at node $n$, time $t$

Imaginary and real part of the load current at node $n$, phase $f$, time $t$

Imaginary and real part of the current demanded by $\mathrm{EV} e$, time $t$

Imaginary and real part of the current generated at node $n$, phase $f$, time $t$

Imaginary and real part of the current in circuit $m n$, phase $f$, time $t$

Imaginary and real part of the current demanded by $\operatorname{ESD} u$, time $t$

Active and reactive power demand at node $n$, phase $f$, time $t$

Active and reactive power generated at node $n$, time $t$

EV $e$ active power consumption, time $t$

Charging and discharging power of the ESD $u$, time $t$
Reactive power delivered by a CB at node $n$, time $t$

Integer variable for the number of steps of the VR in circuit $m n$, phase $f$, time $t$

Imaginary and real part of the voltage at node $n$, phase $f$, time $t$

Real part of the VR voltage calculation auxiliary variable in circuit $m n$, phase $f$, time $t$, position $k$

Binary variable associated with the charging state of $\mathrm{EV} e$, time $t$

\section{INTRODUCTION}

NCREASING energy efficiency and reducing energy costs through a Volt VAr control (VVC) in electrical distribution networks (EDNs) has received plenty of attention in recent years [1]-[4]. The VVC problem finds a control scheme for the proper operation of the devices in the EDN that manage variations in voltage magnitudes and reactive power flows. These devices include among others, voltage regulators (VRs), capacitor banks (CBs) and on-load tap changers (OLTCs) [5]-[8]. Recently, several VVC methodologies have been presented in the specialized literature. In [1], it is proposed a centralized function for VVC, in which, a state estimation based on pseudo-measurements using neural networks is used as input for the VVC. A multiobjective optimization for the VVC was presented in [2]. In this work, a strategy was formulated based on an hourly load forecast for the next day, taking into account the active power demand reduction and the voltage magnitude deviation. Finally, a deterministic framework based on a mixed-integer quadratically constrained programming problem capable of optimally controlling the Volt-VAr problem was formulated in [3].

In recent years, energy storage devices (ESDs) have emerged as a solution to even out the power mismatch between renewable power generators and consumption. The ESDs store the surplus of power for use during time periods of low power generation. Nevertheless, the performance of ESDs has also proven advantageous in improving the economic and technical operation of the EDN [9]-[11]. Reference [9] discussed how the proper functioning of the ESDs in the EDN can improve the economic use of the existing generation, transmission and distribution infrastructure. A mixed-integer second-order cone programming model was presented in [10] to solve the optimal operation problem of ESDs in a radial EDN. The presented approach was tested in two single-phase systems and no dynamic control was presented for ESD charging. In [11], a demand-side management approach was proposed through a day-ahead optimization process for ESDs and distributed generation (DG). This formulation only considered end users, disregarding the economic and operational constraints.

Lately, the number of electric vehicles (EVs) connected to EDNs has received more attention. Because the EVs use the energy from the grid to charge their batteries, a high penetration of EVs can cause the EDN to work outside of acceptable operational limits [12]. The development of EV charging schedules to prevent issues such as overloads and voltage limit violations 
is essential [13]. Several works have approached the EV charging coordination (EVCC) problem [13]-[16]. In [13], an EVCC problem approach was presented. The formulation could properly handle large populations and the random arrival of EVs, but the impact of the EVs on the grid was not considered. A mathematical model for EV integration into the grid was presented in [14]. An economical evaluation was assessed in order to find the optimal energy cost that would benefit both EV owners and the EDN operator. In [15], a multi-period optimization for EV charging in EDNs was presented. The optimization aimed at minimizing the cost of charging EVs. An algorithm based on sensitivities was proposed for the real-time EVCC in [16], considering the random arrivals/departures of EVs, voltage profiles, and power generation limits in order to minimize the total energy cost.

The presented approach overcomes the shortcomings of previous studies, which solved the VVC and the EVCC separately, by engaging both problems in a more global control optimization of the EDN. Furthermore, the advantages offered by ESDs and dispatchable DG units are also considered in this formulation. While VVC devices and ESDs improve the operation of the EDN, the formulation seeks to avoid violations of operational limits and reduce the overall costs in the grid. In addition, the EVCC tries to optimally charge the EVs plugged into the EDN, while avoiding the issues that a high EV penetration may cause.

This work proposes an approach that simultaneously uses the VVC (management of OLTCs, VRs, and CBs), ESD operation, and dispatchable DG units to improve the EVCC in a three-phase unbalanced EDN. Different from the single phase equivalent, the three-phase unbalance representation allows the inclusion of the mutual coupling effects in the circuits, reaching a more realistic representation. Moreover, in order to develop a thorough analysis and a more accurate economic improvement, the load's voltage dependency is also taken into account.

In order to integrate the described devices and achieve the aforementioned objectives, a mixed-integer nonlinear programming (MINLP) formulation is found. Linearization techniques are later used to obtain a mixed-integer linear programming (MILP) model that can be solved using commercial software, guaranteeing the optimal solution to the problem. A step-by-step methodology that offers a broad view of the EDN's behavior in future periods is used to solve a $24 \mathrm{~h}$ time period. The proposed formulation was tested in an EDN with 34 medium-voltage nodes and 144 low-voltage nodes. The results demonstrate the efficiency and robustness of the methodology. This work's main contributions are as follows:

1) A step-by-step methodology that solves the EVCC problem and finds the optimal operation of the EDN, throughout a centralized coordination which takes into account the benefits and flexibility brought to the grid by VVC devices, ESDs, and dispatchable DG units.

2) A multi-period MILP formulation to solve the EVCC problem together with VVC, ESD operation and dispatchable DG units in a three-phase unbalanced EDN, in which loads are represented via a voltage dependent load model.

\section{Problem Formulation and Solution Technigue}

This section describes the EVCC problem as well as the advantages and all technical concerns related to the VVC and the ESD operation. A step-by-step methodology proposed to solve the EVCC, taking into account VVC, ESD operation, and dispatchable DG units in a three-phase unbalanced EDS and using a voltage dependent load model, will be presented.

\section{A. EVCC, VVC and ESD scheduling}

1) EVCC: The EVCC problem aims to find an optimal schedule for the battery charging of the EVs connected into the EDN in a specific time period. This charging scheduling not only has to satisfy the EV owner needs, but also must avoid technical limit violations due to excessive EV load connected into the grid. An improvement in the EVCC can lead to: An economic betterment in the operation of the system, i.e., reduction in energy losses; an amelioration in the fulfillment of technical limits; a cost reduction in the EV recharge; or a reduction in the energy curtailment of the EVs at departure.

In order to find a solution for the EVCC problem, the following considerations are assumed:

1) The initial SOC of every vehicle is known when the vehicle is plugged into the grid.

2) The batteries can be controlled in each time interval into which the time period is divided.

3) The EV user may provide a departure time when the vehicle is plugged; otherwise, it will be assumed that the vehicle will remain plugged in until a probable time interval, determined by the historical of departure of the EV.

2) $V V C$ : The VVC is defined as a control strategy to manage the voltage magnitudes and the reactive power flow throughout the EDN. This management consists of periodic adjustments over devices that inject reactive power into the grid to improve the voltage drop and devices that directly control voltage magnitude [7], [8]. In general, the VVC defines control actions in OLTCs, VRs, and fixed-switchable CBs.

To properly carry out the adjustment over VVC devices, the control actions might be handled by a managed operation. This operation often follows local decision-making based on local measurements of the EDN, i.e., each device is controlled independently. However, each action affects the EDN operation and the performance of other devices. Thus, an integrated coordination of all VVC devices, based on remote measurements on the EDN and operator knowledge, is necessary to obtain an optimal EDN operation through VVC. For centralized coordination, a communication infrastructure and an optimized decision-making algorithm is required to achieve an optimal integrated VVC [17].

Keeping voltage magnitudes inside acceptable limits while optimizing energy loss reduction has been the classical approach when solving the VVC problem. Nonetheless, in recent works the VVC is also used to adjust the voltage profile to manage total demand, since a reduction in voltage magnitude levels can represent a decrease in power consumption [2]. Therefore, considering the effects of special loads in the EDN operation, the 


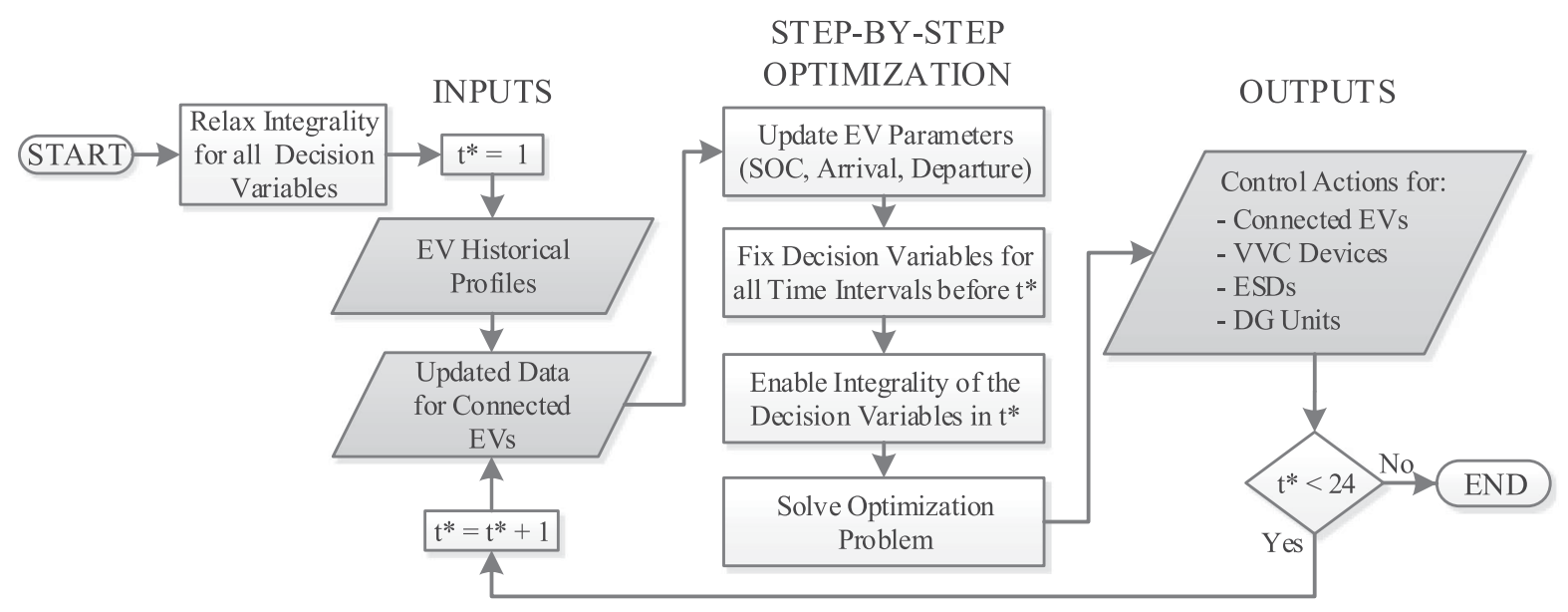

Fig. 1. Step-by-step flowchart of the proposed methodology.

VVC can be a useful tool to improve the solution of the new challenges emerging in the EDN operation. Hence, an optimized dynamic coordination of the grid can be obtained with the inclusion of the VVC, allowing a more flexible adjustment of the $\mathrm{EDN}$, while avoiding voltage limits breaches in the presence of special loads such as EVs.

3) ESD Operation: As mentioned, the solution of the proposed methodology must define an optimal charging agenda for the ESDs. For the ESDs, the charging schedule is responsible for throttling the battery charge and for allowing the EDN to use the stored energy. Thus, the formulation must determine the optimal schedule to charge and discharge the ESDs at each time interval. It is assumed that:

1) The ESDs cannot be discharged beyond their specified Depth of Discharge (DoD).

2) The ESDs can be controlled through communication devices in order to define the charging state at each time step.

3) For each ESD, a goal is defined for the SOC at the end of the time period (e.g., same as the initial SOC). This goal is used to represent the utilization of the ESDs after the considered time period (e.g., one day ahead).

\section{B. Voltage Dependent Load Model}

The demand of an EDN is classified into loads that can be represented as: a) constant power loads, b) constant impedance loads, c) constant current loads, or d) a combination of these. In a VVC framework for EDNs, the load's voltage dependency is a key aspect of the suitable representation of the network operation. As discussed in [2], [3], and [18], VVC models are highly dependent on the accuracy of the load representation. Dependence on the voltage magnitude and frequency is considered in the load models; mathematically, this dependence can be represented by static and dynamic load models described by the traditional ZIP model. For this analysis, two static models are commonly studied: the polynomial load model and the exponential load model [19]. The exponential expression of the load's sensitivity to the voltage and frequency variation is represented by (1) and (2).

$$
\begin{aligned}
P^{D} & =P^{o}\left(\frac{V}{V_{o}}\right)^{\alpha}\left(1+K_{p f}\left(f_{r}-f_{r}^{o}\right)\right) \\
Q^{D} & =Q^{o}\left(\frac{V}{V_{o}}\right)^{\beta}\left(1+K_{q f}\left(f_{r}-f_{r}^{o}\right)\right)
\end{aligned}
$$

where, $f_{r}$ represents the frequency of the bus voltage and $f_{r}^{o}$ represents the nominal frequency. The coefficients $K_{p f}$ and $K_{q f}$ are the frequency sensitivities for the active and reactive power loads, respectively. In this work, the effects associated with frequency are disregarded. Furthermore, with an appropriate adjustment of the constants $\alpha$ and $\beta$ the model can be restricted to the steady-state analysis case (i.e., dependence directly on the voltage magnitude). Appropriate values for these constants may be found in previous works, such as [20].

\section{Methodology}

The proposed methodology defines the status of the control devices (VRs, and CBs), as well as the charging status of the EVs in the network by solving an optimization problem. The operation of the EDN is modeled considering a given horizon (e.g., one-day), which is divided in time intervals. The time duration for every time interval is represented by $\Delta_{t}$. The set $T$ orders time intervals into which the time period is divided.

As shown in Fig. 1, the proposed MILP model is solved at the beginning of each time interval considering the actual number of connected EVs; besides, a forecast for the parameters related to possible EV plugs, such as estimated arrival times and initial state of charge (SOCs), is also taken into account. A dynamic scheduling (rolling multi-period optimization for the EVCC) is performed determining at each time interval the optimal charging agenda of the EVs plugged and to be plugged into the grid. This dynamic scheduling is a part of the step-bystep methodology proposed, which will also determine at each time interval the control scheme for VVC devices, DG units and ESDs on the remaining time period. When a forecasted EV is actually plugged in, the parameters are updated. Nevertheless, if an EV does not arrive during the estimated time interval, a 
different estimation may be used or the EV may be disregarded altogether. It should be noted, that the EVs may arrive and depart during any time interval of the time period. Therefore, the EV's charging agenda will be generated between arrival and departure, ideally dispatching a fully charged battery for every vehicle. Different techniques may be applied to estimate the arrivals, departures, and initial SOCs of the EVs, as presented in [22]-[24].

Operational constraints such as voltage limits, active and reactive power generation limits, and maximum current limits, are taken into account in the model. This way, a step-by-step solution is constructed over the entire time period. The solution at each time interval will determine the charging state for the EVs and ESDs, the energy delivered by the DG units, the number of modules operating in each $\mathrm{CB}$, and the tap position for each phase of every VR. The nature of the control variables associated with each device is binary, which makes the VCC problem hard to solve. In order to reduce the computational complexity, only the control variables for the current time interval are treated as binary variables, i.e., control variables for the remaining time intervals are relaxed. This strategy makes it possible to solve the EVCC problem with reduced computational effort, obtaining the solution for the current time interval using updated information. An important upside of this methodology is that it offers a broad view of the state of the EDN throughout the whole time period.

\section{Mixed-Integer Nonlinear Programming Model for EVCC}

A mixed integer nonlinear programming (MINLP) model is presented in (3)-(38) for the proposed EVCC problem. The solution found by the formulation must define: the power delivered by each dispatchable DG unit $\left(P_{n, t}^{G}\right)$, the number of modules connected in each CB $\left(B_{n, t}\right)$, the tap position for each $\operatorname{VR}\left(t p_{m n, f, t}\right)$, the charging schedule for each $\operatorname{EV}\left(y_{n, t}\right)$, and the charging and discharging schedule for each $\operatorname{ESD}\left(P_{u}^{S D+}\right.$ and $P_{u}^{S D-}$, respectively).

1) Objective Function (OF): Represented by (3), the OF aims to minimize the cost of the energy provided by the substation (SE) (first term), the DG energy cost (second term), the cost of the energy curtailment on EVs (third term), and the cost of the energy injected from the ESDs to the grid (fourth term), and to penalize the deviation from the defined goals for the SOC of the ESDs (fifth term).

$$
\begin{aligned}
& \min \sum_{f \in F} \sum_{t \in T} \zeta_{S E, t}^{G} \Delta_{t}\left(V_{S E, f, t}^{r e} I_{S E, f, t}^{G r e}+V_{S E, f, t}^{i m} I_{S E, f, t}^{G i m}\right) \\
& +\sum_{n \in N} \sum_{t \in T} \zeta_{n, t}^{G} \Delta_{t} P_{n, t}^{G}+\sum_{e \in \Xi} \zeta_{c}^{E V} E_{e}^{E V c} \\
& +\sum_{u \in S D} \sum_{t \in T} \zeta^{S D} \Delta_{t} P_{n, t}^{S D-}+\sum_{u \in S D} \zeta_{c}^{S D} E_{u}^{S D c}
\end{aligned}
$$

2) Load Flow Constraints: The equations (4)-(13) model the steady-state operation of a three-phase unbalanced EDN considering the presence of DG units (set $D G$ ), CBs (set $C B$ ),
VRs (set $V R$ ), ESDs (set $S D$ ) and EVs (set $\Xi)$.

$$
\begin{aligned}
I_{n, f, t}^{G r e}+ & \sum_{n \in C B} I_{n, d}^{c b r e}+\sum_{k m \in L \cup V R} I_{k m, f, t}^{r e}-\sum_{m n \in L} I_{m n, t}^{r e} \\
& -\left(\sum_{k m \in L} B_{k m, f}^{s h}+\sum_{m n \in L} B_{m n, f}^{s h}\right) \frac{V_{m, f, t}^{i m}}{2}=I_{m, f, t}^{D r e} \\
& +\sum_{e \in \Xi} I_{e, t}^{E V r e} \gamma(e, m, f)+\sum_{u \in S D} I_{u, t}^{S D r e} \gamma(u, m, f)
\end{aligned}
$$

$$
\begin{aligned}
I_{n, f, t}^{\text {Gim }}+ & \sum_{n \in C B} I_{n, d}^{c b i m}+\sum_{k m \in L \cup V R} I_{k m, f, t}^{i m}-\sum_{m n \in L} I_{m n, t}^{i m} \\
& -\left(\sum_{k m \in L} B_{k m, f}^{s h}+\sum_{m n \in L} B_{m n, f}^{s h}\right) \frac{V_{m, f, t}^{r e}}{2}=I_{m, f, t}^{D i m} \\
& +\sum_{e \in \Xi} I_{e, t}^{E V i m} \gamma(e, m, f)+\sum_{u \in S D} I_{u, t}^{S D i m} \gamma(u, m, f)
\end{aligned}
$$

$I_{n, f, t}^{D r e}=\left(P_{n, f, t}^{D} V_{n, f, t}^{r e}+Q_{n, f, t}^{D} V_{n, f, t}^{i m}\right) / V_{n, f, t}^{r e^{2}}+V_{n, f, t}^{i m^{2}}$

$I_{n, f, t}^{D i m}=\left(P_{n, f, t}^{D} V_{n, f, t}^{i m}-Q_{n, f, t}^{D} V_{n, f, t}^{r e}\right) / V_{n, f, t}^{r e^{2}}+V_{n, f, t}^{i m^{2}}$

$P_{n, f, t}^{D}=P_{n, f, t}^{o}\left(\sqrt{V_{n, f, t}^{r e^{2}}+V_{n, f, t}^{i m^{2}}} / V_{o}\right)^{\alpha_{n, f}}$

$Q_{n, f, t}^{D}=Q_{n, f, t}^{o}\left(\sqrt{V_{n, f, t}^{r e^{2}}+V_{n, f, t}^{i m^{2}}} / V_{o}\right)^{\beta_{n, f}}$

$V_{m, f, t}^{r e}-V_{n, f, t}^{r e}=\sum_{h \in F}\left(R_{m n, f, h} I_{m n, h, t}^{r e}-X_{m n, f, h} I_{m n, h, t}^{i m}\right)$

$V_{m, f, t}^{i m}-V_{n, f, t}^{i m}=\sum_{h \in F}\left(X_{m n, f, h} I_{m n, h, t}^{r e}+R_{m n, f, h} I_{m n, h, t}^{i m}\right)$

$\underline{V}^{2} \leq V_{n, f, t}^{r e}{ }^{2}+V_{n, f, t}^{i m}{ }^{2} \leq \bar{V}^{2}$

$0 \leq I_{m n, f, t}^{r e}+I_{m n, f, t}^{i m}{ }^{2} \leq \bar{I}_{m n}{ }^{2}$

$\forall m n \in L, \forall n \in N, f \in F, t \in T$

where, $F, L$, and $N$, are the sets of phases, circuits and nodes, respectively. The balance of the currents at each node is represented by (4) and (5). Moreover, $\gamma$ is a binary function that takes a value of 1 if the EV $e$ or ESD $u$ are connected at node $m$ and at phase $f$. The load currents shown in (6) and (7) define the relationship between voltage, current and the active and reactive powers demanded by the loads. Moreover, (8) and (9) are the exponential representation of the load's voltage dependency. Equations (10) and (11) are the result of applying Kirchhoff's Voltage Law to each independent loop of the EDN. Finally, the limits for the voltage magnitude and current capacity in each circuit are stated in (12) and (13), respectively. 
3) DG Constraints: The operation limits of the DG units are defined by (14)-(16), while the active and reactive power injections are represented by (17)-(18), respectively.

$$
\begin{aligned}
& 0 \leq P_{n, t}^{G} \leq \bar{P}_{n}^{G} \\
& \underline{Q}_{n}^{G} \leq Q_{n, t}^{G} \leq \bar{Q}_{n}^{G} \\
& \left|Q_{n, t}^{G}\right| \leq P_{n, t}^{G} \tan \left(\arccos \left(\phi_{n}\right)\right) \\
& P_{n, t}^{G} / 3=V_{n, f, t}^{r e} I_{n, f, t}^{G r e}+V_{n, f, t}^{i m} I_{n, f, t}^{G i m} \\
& Q_{n, t}^{G} / 3=-V_{n, f, t}^{r e} I_{n, f, t}^{G i m}+V_{n, f, t}^{i m} I_{n, f, t}^{G r e} \\
& \left.\quad \forall n \in N\right|_{n \exists D G}, f \in F, t \in T
\end{aligned}
$$

4) CBs Constraints: Similar to the DG units, the active and reactive power of the switchable CBs are represented by (19) and (20). The value for the active power injection of every CB will always be equal to zero. Equation (21) represents the number of capacitor modules connected to the grid for each bank, while the allowable limit of modules is modeled by (22). Finally, as the lifetime of switchable CBs is significantly impacted by the number of operations (23), this limits the maximum number of operations permitted over the entire time period.

$$
\begin{aligned}
& 0=V_{n, f, t}^{r e} I_{n, f, t}^{c b r e}+V_{n, f, t}^{i m} c_{n, f, t}^{c b i m} \\
& Q_{n, t}^{c b} / 3=-V_{n, f, t}^{r e} I_{n, f, t}^{c b i m}+V_{n, f, t}^{i m} I_{n, f, t}^{c b r e} \\
& Q_{n, t}^{c b}=B_{n, t} Q_{n}^{e s p} \\
& 0 \leq B_{n, t} \leq \bar{B}_{n} \\
& \sum_{t \in T}\left|B_{n, t}-B_{n, t-1}\right| \leq \Delta^{c b} \\
& \left.\quad \forall n \in N\right|_{n \exists C B}, f \in F, t \in T
\end{aligned}
$$

5) VR and OLTC Constraints: The mathematical model of a VR or a OLTC is shown in (24)-(27). In this model, (24) represents the real regulated voltage and (25) represents the real regulated current on each VR/OLTC. Expression (26) represents the minimum and maximum limits of the tap position represented by the integer variable $t p_{m n, f, d}$. Analogue to (23), (27) limits the maximum number of operations allowable for a VR/OLTC over the time period in order to avoid excessive wear and tear of these devices. Expressions similar to (24) and (25) were implemented to describe the imaginary component for both the regulated voltage and current.

$$
\begin{gathered}
V_{n, f, t}^{r e}=\left(1+\% R_{m n} t p_{m n, f, t} / T p_{m n}\right) V_{m, f, t}^{r e} \\
I_{k m, f, t}^{r e}=\left(1+\% R_{m n} t p_{m n, f, t} / T p_{m n}\right) I_{m n, f, t}^{r e} \\
-T p_{m n} \leq t p_{m n, f, t} \leq T p_{m n} \\
\sum_{t \in T}\left|t p_{m n, f, t}-t p_{m n, f, t-1}\right| \leq \Delta^{v r} \\
\left.\forall m n \in L\right|_{m n \exists V R}, f \in F, t \in T
\end{gathered}
$$

6) ESD Constraints: For the ESDs, (28) and (29) represent the active and reactive power exchange between each ESD and the grid. In (30), the energy balance in an ESD is written in terms of the energy that must be charged, the charging power in each time interval and the defined goal for the SOC at the end of the charging period. Equation (31) defines the energy stored in each SD at every interval and (32) limits the ESD's energy level ensuring that it does not exceed the battery's maximum capacity or the pre-established DoD. Finally, (33) defines the limits for the charging and discharging active powers for each ESD.

$$
\begin{aligned}
& \left(P_{u, t}^{S D+}-P_{u, t}^{S D-}\right) / 3=V_{u, t}^{r e} I_{u, t}^{S D r e}+V_{u, t}^{i m} I_{u, t}^{S D i m} \\
& 0=-V_{u, t}^{r e} I_{u, t}^{S D i m}+V_{u, t}^{i m} I_{u, t}^{S D r e} \\
& E_{u}^{S D_{f}}=E_{u, T}^{S D}+E_{u}^{S D c} \\
& E_{u, t}^{S D}=E_{u}^{S D i}+\sum_{k \in T, k \leq t} \Delta_{k}\left(P_{u, t}^{S D+} \eta_{u}^{+}-P_{u, t}^{S D-} / \eta_{u}^{-}\right) \\
& \min \left(E_{u}^{S D i}, \bar{E}_{u}^{S D} D o D\right) \leq E_{u, t}^{S D} \leq \bar{E}_{u}^{S D} \\
& 0 \leq P_{u, t}^{S D+}, P_{u, t}^{S D-} \leq \bar{P}_{u}^{S D} \quad \forall u \in S D, t \in T
\end{aligned}
$$

7) EV Constraints: The active and reactive powers of the EVs are presented in (34) and (35), respectively. The instant power in (36) is represented in terms of the maximum charging power and the binary control variable $y_{n, t}$. Constraint (37) determines the energy curtailment on each EV, as (38) determines the energy of each EV at departure. The variable $y_{n, t}$ has a value of 1 if the EV battery is charging at its maximum power $\bar{P}_{e}^{E V c h}$ and a value of 0 otherwise.

$$
\begin{aligned}
P_{e, t}^{E V} & =V_{e, t}^{r e} I_{e, t}^{E V r e}+V_{e, t}^{i m} I_{e, t}^{E V i m} \\
0 & =-V_{e, t}^{r e} I_{e, t}^{E V i m}+V_{e, t}^{i m} I_{e, t}^{E V r e} \\
P_{e, t}^{E V} & =\bar{P}_{e}^{E V c h} y_{e, t} \\
\bar{E}_{e}^{E V} & =E_{e}^{E V}+E_{e}^{E V c} \\
E_{e}^{E V} & =E_{e}^{i n i}+\sum_{t \in T} \Delta_{t} P_{e, t}^{E V} \eta_{e} \quad \forall e \in \Xi, t \in T
\end{aligned}
$$

\section{E. Mixed-Integer Linear Programming Model for EVCC}

To enhance the robustness of the solution and to enable the utilization of classic optimization methods and commercial solvers, linearization techniques are applied to the nonlinear expressions in (3)-(38) in order to obtain a MILP formulation.

1) Linearization for Power Flow Nonlinear Constraints: Constraints (6)-(9), (12), and (13), are nonlinear expressions. Equations (6)-(9) are rewritten as shown in (39) and (40). Constraints (41) and (42) show the first-order linearization for (39) and (40) around an estimated operation point $\left(V_{n, f, t}^{r e^{*}}, V_{n, f, t}^{i m^{*}}\right)$. Furthermore, (12) and (13) are linearized as presented in [21].

$$
\begin{aligned}
g\left(V_{n, f, t}^{r e}, V_{n, f, t}^{i m}\right)= & P_{n, f, t}^{o} \frac{V_{n, f, t}^{r e}}{V_{o}^{\alpha_{n, f}}}\left(V_{n, f, t}^{r e^{2}}+V_{n, f, t}^{i m^{2}}\right)^{\frac{\alpha_{n, f}}{2}-1} \\
& +Q_{n, f, t}^{o} \frac{V_{n, f, t}^{i m}}{V_{o}^{\beta_{n, f}}}\left(V_{n, f, t}^{r e^{2}}+V_{n, f, t}^{i m^{2}}\right)^{\frac{\beta_{n, f}}{2}-1}
\end{aligned}
$$




$$
\begin{gathered}
h\left(V_{n, f, t}^{r e}, V_{n, f, t}^{i m}\right)=P_{n, f, t}^{o} \frac{V_{n, f, t}^{i m}}{V_{o}^{\alpha_{n, f}}}\left(V_{n, f, t}^{r e^{2}}+V_{n, f, t}^{i m^{2}}\right)^{\frac{\alpha_{n, f}}{2}-1} \\
-Q_{n, f, t}^{o} \frac{V_{n, f, t}^{r e}}{V_{o}^{\beta_{n, f}}}\left(V_{n, f, t}^{r e^{2}}+V_{n, f, t}^{i m^{2}}\right)^{\frac{\beta_{n, f}}{2}-1} \\
I_{n, f, t}^{D r e}=g^{*}+\left.\frac{\partial g}{\partial V^{r e}}\right|^{*}\left(V_{n, f, t}^{r e}-V_{n, f, t}^{r e^{*}}\right)+\left.\frac{\partial g}{\partial V^{i m}}\right|^{*} \\
\left(V_{n, f, t}^{i m}-V_{n, f, t}^{i m^{*}}\right) \\
I_{n, f, t}^{D i m}=h^{*}+\left.\frac{\partial h}{\partial V^{r e}}\right|^{*}\left(V_{n, f, t}^{r e}-V_{n, f, t}^{r e^{*}}\right)+\left.\frac{\partial h}{\partial V^{i m}}\right|^{*} \\
\left(V_{n, f, t}^{i m}-V_{n, f, t}^{i m^{*}}\right) \\
\forall n \in N, f \in F, t \in T
\end{gathered}
$$

2) Linearization of DG, CBs, ESDs and EVs Nonlinear Constraints: The nonlinear expressions of the DG, CBs, ESDs and EVs (constraints (17), (18), (19), (20), (28), (29), (34) and (35)) are approximated using an estimated operation point $\left(V_{n, f, t}^{r e e^{*}}, V_{n, f, t}^{i m^{*}}\right)$ as shown in [21]. The quality of the estimated operation point will define the approximation error. Historical data and the EDN operator knowledge, nominal values for the voltage magnitudes, or a linear relaxation of the MILP model initially solved (in which the binary nature of the decision variables is temporarily ignored), could be used to estimate the operation point.

3) Linearization of VR/OLTC Nonlinear Constraints: In the expressions (24) and (25), the product of the decision variables $t p_{m n, f, t}$ and, $V_{m, f, t}$ or $I_{m n, f, t}$ on the real and imaginary components is linearized. To obtain linear expressions, the integer number of steps is represented as a set of binary variables $b t_{m n, f, t}$ and the products $t p_{m n, f, t} V_{m, f, t}, t p_{m n, f, t} I_{m n, f, t}$ are substituted by the variables $V_{m n, f, t, k}^{c}$ and $I_{m n, f, t, k}^{c}$, respectively, on the real and imaginary components.

$$
\begin{gathered}
V_{n, f, t}^{r e}=\left(1-\% R_{m n}\right) V_{m, f, t}^{r e}+\sum_{k=1}^{2 T p_{m} n} \frac{\% R_{m n}}{T p_{m n}} V_{m n, f, t, k}^{c(r e)} \\
I_{k m, f, t}^{r e}=\left(1-\% R_{m n}\right) I_{m n, f, t}^{r e}+\sum_{k=1}^{2 T p_{m n}} \frac{\% R_{m n}}{T p_{m n}} I_{m n, f, t, k}^{c(r e)} \\
\sum_{k=1}^{2 T p_{m n}} b t_{m n, f, t, k}-T p_{m n}=t p_{m n, f, t} \\
\left|V_{m, f, t}^{r e}-V_{m n, f, t, k}^{c(r e)}\right| \leq \bar{V}\left(1-b t_{m n, f, t, k}\right) \\
\left|V_{m n, f, t, k}^{c(r e)}\right| \leq \bar{V} b t_{m n, f, t, k} \\
\left|I_{m n, f, t}^{r e}-I_{m n, f, t, k}^{c(r e)}\right| \leq \bar{I}_{m n}\left(1-b t_{m n, f, t, k}\right) \\
\left|I_{m n, f, t, k}^{c(r e)}\right| \leq \bar{I}_{m n} b t_{m n, f, t, k} \\
b t_{m n, f, t, k} \leq b t_{m n, f, t, k-1}
\end{gathered}
$$

$\left.\forall m n \in L\right|_{m n \exists V R}, f \in F, t \in T, k \in 1 . .2 T p_{m n}$

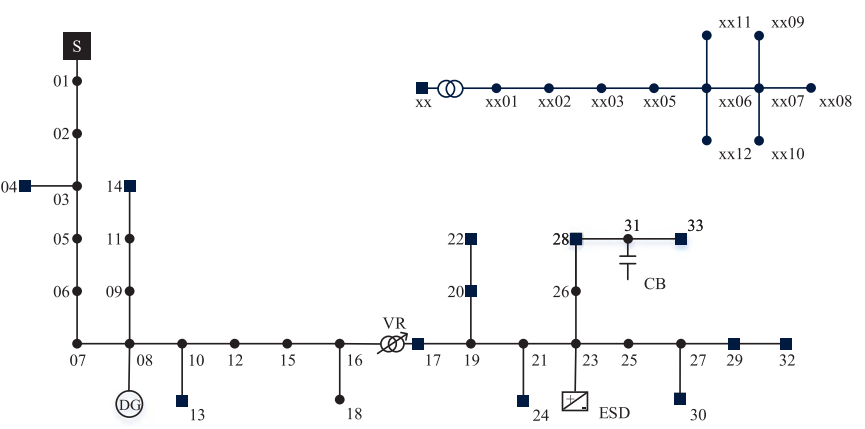

Fig. 2. 178-nodes test system.

Expressions (43)-(50) are linear extensions of (24)-(27), where (43) represents the calculation of the regulated voltage and (44) represents the calculation of the regulated current for the real component. Equation (45) associates the binary variable with the tap integer variable. Constraint (46) defines the auxiliary variable $V_{m n, f, d, k}^{c}$ and (47) describes its limits. In the same way, (48) defines the auxiliary variable $I_{m n, f, d, k}^{c}$ and (49) describes its limits. Expression (50) represents the sequencing of the binary variable $b t_{m n, f, d}$ in the previous tap position. For time intervals in which the binary nature of the control variables is disregarded, the equations above are not suitable. Therefore, (51) and (52) are used to calculate the relaxed value of $b t$ for each VR/OLTC.

In other words, (43)-(50) are used to calculate the tap position in the actual time interval and, (50), (51) and (52) are constraints applied to the rest of the time period. Similar to the approximated values of the voltages, the quality of the approximated values of the currents $\left(I_{m n, f, t}^{r e^{*}}\right.$ and $\left.I_{m n, f, t}^{i m^{*}}\right)$ depends on the EDN operator's knowledge. Expressions similar to (43)-(52) were implemented to linearize the imaginary component.

$$
\begin{aligned}
& V_{n, f, t}^{r e}=\left(1-\% R_{m n}\right) V_{m, f, t}^{r e}+\sum_{k=1}^{2 T p_{m n}} b t_{m n, f, t, k} \frac{\% R_{m n}}{T p_{m n}} V_{n, f, t}^{r e^{*}} \\
& I_{k m, f, t}^{r e}=\left(1-\% R_{m n}\right) I_{m n, f, t}^{r e}+\sum_{k=1}^{2 T p_{m n}} b t_{m n, f, t, k} \frac{\% R_{m n}}{T p_{m n}} I_{m n, f, t}^{r e^{*}}
\end{aligned}
$$

$$
\left.\forall m n \in L\right|_{m n \exists V R}, f \in F, t \in T
$$

\section{TESTS AND RESUltS}

To assess the proposed methodology, tests were carried out on a three-phase EDN with 34 nodes at medium voltage $(24.9 \mathrm{kV})$ and 144 nodes at low voltage ( $480 \mathrm{~V}$ ) shown in Fig. 2. The EDN was adapted from the IEEE 34-node test system; for further information, the complete description of the EDN used is available in [25]. The test system includes a DG unit at node 8, an ESD at node 23, a VR connected at the end-point of circuit 16-17 and a $\mathrm{CB}$ at node 31 . Moreover, phases $A, B$, and $C$ were loaded with $33 \%, 35 \%$, and $32 \%$ of the total demand, respectively. The values of the parameters $\alpha$ and $\beta$, used to represent different types of loads in the voltage dependent model, are presented 
TABLE I

HOURLY ENERGY COSTS AND LOAD VARIATIONS

\begin{tabular}{lccccc}
\hline \hline Hour & $\begin{array}{c}\text { Energy cost } \\
(\$ / \mathrm{kWh})\end{array}$ & $\begin{array}{c}\text { \% of peak } \\
\text { load }\end{array}$ & Hour & $\begin{array}{c}\text { Energy cost } \\
(\$ / \mathrm{kWh})\end{array}$ & $\begin{array}{c}\text { \% of peak } \\
\text { load }\end{array}$ \\
\hline $08: 00$ & 0.0442 & 50 & $\mathbf{2 0 : 0 0}$ & $\mathbf{0 . 0 8 7 5}$ & $\mathbf{8 9}$ \\
$09: 00$ & 0.0529 & 55 & $\mathbf{2 1 : 0 0}$ & $\mathbf{0 . 0 8 3 7}$ & $\mathbf{8 7}$ \\
$10: 00$ & 0.0606 & 63 & $\mathbf{2 2 : 0 0}$ & $\mathbf{0 . 0 7 2 1}$ & $\mathbf{7 5}$ \\
$11: 00$ & 0.0673 & 70 & $\mathbf{2 3 : 0 0}$ & $\mathbf{0 . 0 6 2 5}$ & $\mathbf{6 9}$ \\
$12: 00$ & 0.0721 & 75 & $\mathbf{0 0 : 0 0}$ & $\mathbf{0 . 0 5 2 9}$ & $\mathbf{5 9}$ \\
$13: 00$ & 0.0798 & 83 & $\mathbf{0 1 : 0 0}$ & $\mathbf{0 . 0 3 8 5}$ & $\mathbf{4 6}$ \\
$14: 00$ & 0.0847 & 88 & $\mathbf{0 2 : 0 0}$ & $\mathbf{0 . 0 3 2 7}$ & $\mathbf{4 4}$ \\
$15: 00$ & 0.0866 & 90 & $\mathbf{0 3 : 0 0}$ & $\mathbf{0 . 0 3 1 7}$ & $\mathbf{4 2}$ \\
$16: 00$ & 0.0914 & 95 & $\mathbf{0 4 : 0 0}$ & $\mathbf{0 . 0 3 3 6}$ & $\mathbf{4 1}$ \\
$17: 00$ & 0.0933 & 97 & $\mathbf{0 5 : 0 0}$ & $\mathbf{0 . 0 3 2 7}$ & $\mathbf{4 0}$ \\
$\mathbf{1 8 : 0 0}$ & $\mathbf{0 . 0 9 6 2}$ & $\mathbf{1 0 0}$ & $\mathbf{0 6 : 0 0}$ & $\mathbf{0 . 0 3 9 4}$ & $\mathbf{4 5}$ \\
$\mathbf{1 9 : 0 0}$ & $\mathbf{0 . 0 8 9 5}$ & $\mathbf{9 1}$ & $\mathbf{0 7 : 0 0}$ & $\mathbf{0 . 0 4 2 3}$ & $\mathbf{4 8}$ \\
\hline \hline
\end{tabular}

in [25]. The hourly energy costs and load variation percentages are shown in Table I. It was assumed that EVs could arrive and depart at any time interval during the period in bold, based on the fact that this is the time window when the EV demand has a greater impact.

The DG unit energy cost $\zeta_{n, t}^{G}$ was equal to $0.045 \mathrm{US} \$ \mathrm{kWh}$, while its maximum active power was $350 \mathrm{~kW}$ and its minimum and maximum reactive powers were equal to $-175 \mathrm{kVAr}$ and $175 \mathrm{kVAr}$, respectively. Finally, the minimum power factor for the operation was 0.90 .

The ESD was assumed to be owned by the EDN operator. Hence, the cost for the active power injected into the grid by the ESD $\zeta^{S D}$ was disregarded. Moreover, the ESD maximum energy capacity was $3 \mathrm{MWh}$, with a maximum charge and discharge power of $300 \mathrm{~kW}, 10 \%$ of DoD , and charging and discharging efficiencies of $100 \%$. The expected ESD SOC at the end of the time period was set at its initial energy level. Finally, the curtailment costs $\zeta_{c}^{S D}$ was set to $100 \mathrm{US} \$ \mathrm{kWh}$.

The VR was assumed to be a three-phase VR built from three single-phase VRs. This feature offers the flexibility of controlling each phase independently. Each single-phase VR could vary the voltage with a regulation ratio of $\pm 10 \%$; in addition, this ratio was equally distributed across 16 tap positions $( \pm 8)$. Furthermore, the switchable CB capacity was $300 \mathrm{kVAr}$, divided into 6 individual modules. For these tests, the maximum number of allowable operations for both CB and VR $\left(\Delta^{c b}\right.$ and $\Delta^{v r}$, respectively) were set at unreachable values, i.e., the number of operations for each device over the time period was unlimited.

In addition, 180 consumers with EVs were considered in the network, representing almost a $40 \%$ penetration. Two types of EVs were considered: the 'Tesla Model S' with a $70 \mathrm{kWh}$ battery capacity and $10 \mathrm{~kW}$ charging power [26], and the 'Nissan Leaf' with a $24 \mathrm{kWh}$ battery capacity and $4 \mathrm{~kW}$ charging power [27]. Moreover, a $100 \%$ efficiency for all EV charging stations was assumed and the curtailment cost for the $\mathrm{EVs} \zeta_{c}^{E V}$ was set to $100 \mathrm{US} \$ / \mathrm{kWh}$.

The proposed MILP model was written in the mathematical language AMPL [28] and solved using the commercial solver CPLEX [29], on a computer with an Intel $i 74770$ processor. Tests were carried out for four different cases with the following control alternatives:
TABLE II

SUMMARY OF THE TEST CASES

\begin{tabular}{lccccc}
\hline \hline & SE & DG & Total \\
Case & $\begin{array}{c}\text { Energy } \\
\text { Cost }(\$)\end{array}$ & $\begin{array}{c}\text { Energy } \\
\text { Cost }(\$)\end{array}$ & $\begin{array}{c}\text { Energy } \\
\text { Cost }(\$)\end{array}$ & $\begin{array}{c}\text { Curtailment } \\
(\mathrm{kWh})\end{array}$ & $\begin{array}{c}\text { Objective } \\
\text { Function } \\
(\$)\end{array}$ \\
\hline I & 1957.69 & 314.98 & 2272.67 & 274 & 29672.67 \\
II & 1969.79 & 336.18 & 2305.97 & 104 & 12705.97 \\
III & 1905.24 & 279.13 & 2184.37 & 0 & 2184.37 \\
IV & 1852.81 & 287.94 & 2140.75 & 0 & 2140.75 \\
\hline \hline
\end{tabular}

1) Case I: ESD not available; VVC predefined.

2) Case II: ESD enabled; VVC predefined.

3) Case III: ESD not available; VVC enabled.

4) Case IV: ESD enabled; VVC enabled.

For the Cases I and II, in which the VVC was predefined, the VVC devices followed preset settings. These settings are defined by the EDN operator following the daily conventional demand pattern.

Table II shows a summary of the results from the test cases. Case I, which includes only the control over the EVs plugged into the system, presented the worst solution, with high EV energy curtailment and expensive overall energy cost. From Case II, it can be seen how the enabling of the ESD in the grid leads to a significant reduction in the EV energy curtailment, hence, an improvement in the EVCC solution. Besides, Case II presented an increment on the overall energy costs due to a higher EV demand attended. Moreover, Case III shows how with the inclusion of the VVC, the grid is able to attend the whole EV demand, avoiding technical limit breaches. Finally, the best OF value with the lowest overall energy cost and no EV energy curtailment was obtained in Case IV, demonstrating the advantages of the proposed control methodology.

The time limit for the solution process at each time interval was $600 \mathrm{~s}$. If the time limit was reached and the optimal solution had not been found, the best integer solution yet found was adopted. Furthermore, it was verified that the gap presented in those time intervals, in which the solver could not find the optimal solution within the specified time limit, never exceeded $0.1 \%$.

Fig. 3 shows the active power injected into the grid by the SE, the DG unit, and the ESD for Cases I-IV, respectively. In addition, this figure shows, for all cases, the power demanded by the EVs and the ESD when charging (by convention, the active power due to these demands is shown in negative values). It should be noted that the methodology found an optimal schedule to recharge the EVs mainly at low-cost energy time intervals. Nevertheless, energy curtailments were presented when the grid was unable to meet the EV demand. When VVC was enabled, the EDN could completely charge the EVs during the low-cost energy time intervals, thereby achieving a better OF. On the other hand, the ESD improves the EVCC by allowing a higher number of EVs to be scheduled at the last time intervals. Furthermore, as the VVC was enabled, the total amount of power demanded in the time period decreased as well as the energy bought from the DGs. 


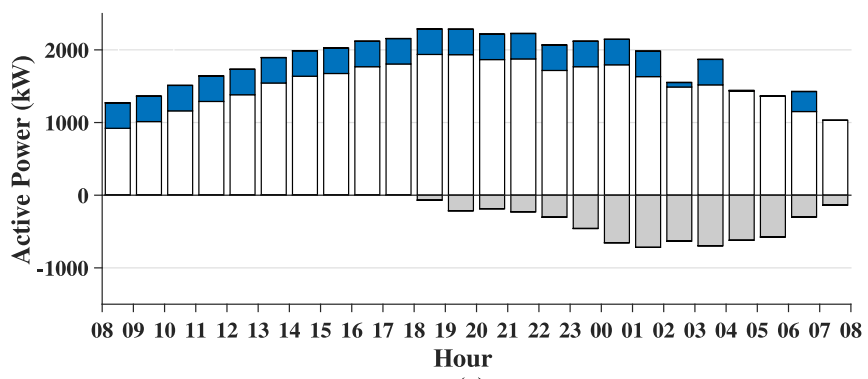

(a)

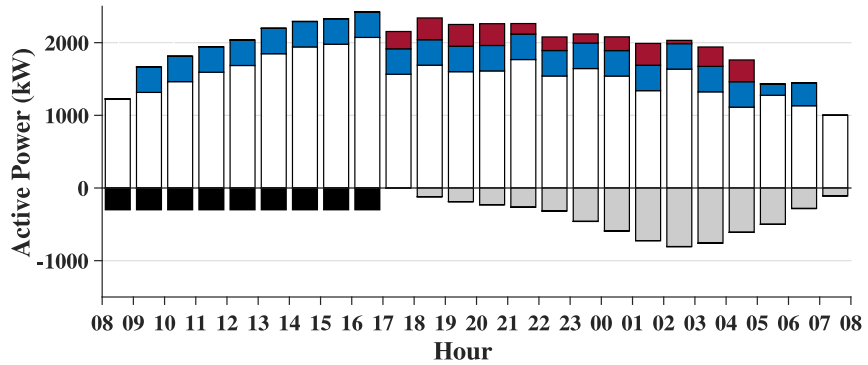

(b)

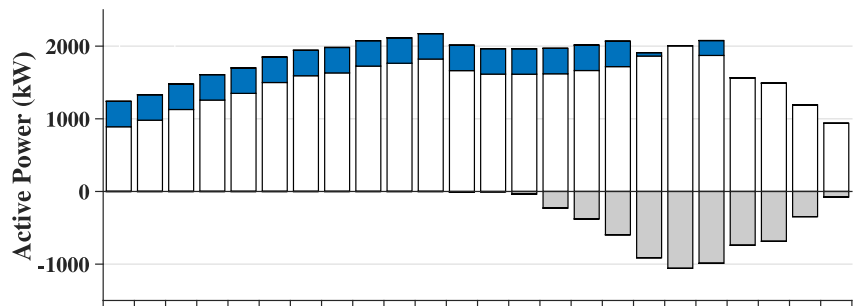

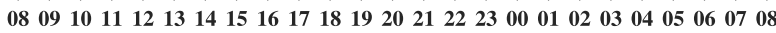
Hour

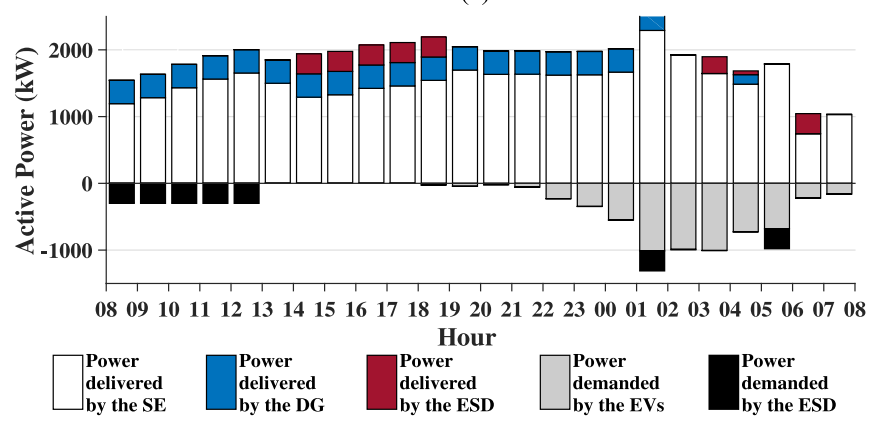

(d)

Fig. 3. Active power injected into the grid for a) Case I, b) Case II, c) Case III and d) Case IV.

The VVC pre-set profile and the VVC schemes for Cases III and IV are shown in Table III. For every case, the VVC seeks to fulfill the technical constraints and determine the best economical operation of the EDN. When enabled, all the modules of the $\mathrm{CB}$ were plugged into the grid; this permits a greater voltage reduction at the VR, therefore, achieving a better economic performance. Although the limit for the number of switching operations was set at a very large number, the number of switching operations for any VVC device over the entire time period for Cases III and IV was below 30.

Fig. 4 shows the minimum voltage magnitude profiles for each time interval in the EDN, evidencing the fulfillment of voltage limits in all cases. Due to the voltage dependency of the loads, in the cases where the VVC was enabled (Cases III
TABLE III

VR TAP POSITION RANGE FOR EACH PHASE AND CB ModUleS

\begin{tabular}{lccccc}
\hline \hline Time intervals & & $07-12$ & $13-21$ & $22-01$ & $02-06$ \\
\hline Predefined & A, B, C & $\{2\}$ & $\{4\}$ & $\{2\}$ & $\{1\}$ \\
Settings & CB & $\{3\}$ & $\{5\}$ & $\{3\}$ & $\{1\}$ \\
\hline \multirow{4}{*}{ Case III } & $A$ & $\{-6,-3\}$ & $\{-3,-1\}$ & $\{-4,4\}$ & $\{-1,4\}$ \\
& $B$ & $\{-4,-2\}$ & $\{-1,1\}$ & $\{2,6\}$ & $\{-5,5\}$ \\
& $C$ & $\{-6,-4\}$ & $\{-3,-2\}$ & $\{-1,0\}$ & $\{-1,5\}$ \\
& CB & $\{6\}$ & $\{6\}$ & $\{6\}$ & $\{6\}$ \\
& $A$ & $\{-5,-3\}$ & $\{-3,-1\}$ & $\{-4,4\}$ & $\{-2,3\}$ \\
\hline Case IV & $B$ & $\{-4,-2\}$ & $\{-1,0\}$ & $\{1,5\}$ & $\{-3,5\}$ \\
& $C$ & $\{-5,-4\}$ & $\{-3,-2\}$ & $\{-1,2\}$ & $\{-6,4\}$ \\
& CB & $\{6\}$ & $\{6\}$ & $\{6\}$ & $\{6\}$ \\
\hline \hline
\end{tabular}

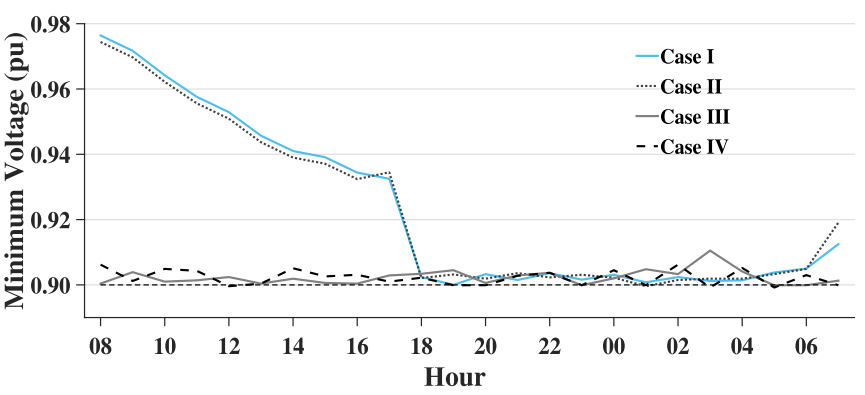

Fig. 4. Minimum voltage profile.

and IV), the minimum voltage profile was kept as close as possible to the lower boundary. This feature can be quantified by the economical operation improvement shown in Table II. Thereby, comparing Cases I and III, there is a reduction in the overall energy cost of the EDN throughout the entire time period, even though the EVs were completely attended only in Case III.

To properly validate the importance of the voltage dependent load model, the formulation was solved considering constant power in the load representation (i.e., $\alpha=0$ and $\beta=0$ ). A power flow considering the voltage dependent load model was later executed, fixing the control variables obtained from the constant power test. The results showed that the solutions obtained using an approach without proper load modeling breached technical limits when applied to the grid. Furthermore, the solution obtained from the constant power test not only exceeded operational constraints, but also presented higher OF.

Finally, in order to evaluate the precision of the proposed formulation, all the decision variables of the solution found by the methodology [i.e., the power delivered by each dispatchable DG unit $\left(P_{n, t}^{G}\right)$; the number of modules connected in each CB $\left(B_{n, t}\right)$; the tap position for each VR $\left(t p_{m n, f, t}\right)$; the charging schedule for each $\mathrm{EV}\left(y_{n, t}\right)$; and the charging and discharging schedule for each $\operatorname{ESD}\left(P_{u}^{S D+}\right.$ and $P_{u}^{S D-}$, respectively)] in Case IV were used to solve a conventional AC power flow. Thereby, for the OF, the error found by comparing the MILP formulation and the conventional power flow was $0.023 \%$. Moreover, the voltage magnitude error percentage obtained for all nodes at the time intervals with the highest conventional load and EV demand (hours 18:00 and 01:00, respectively), is presented in 


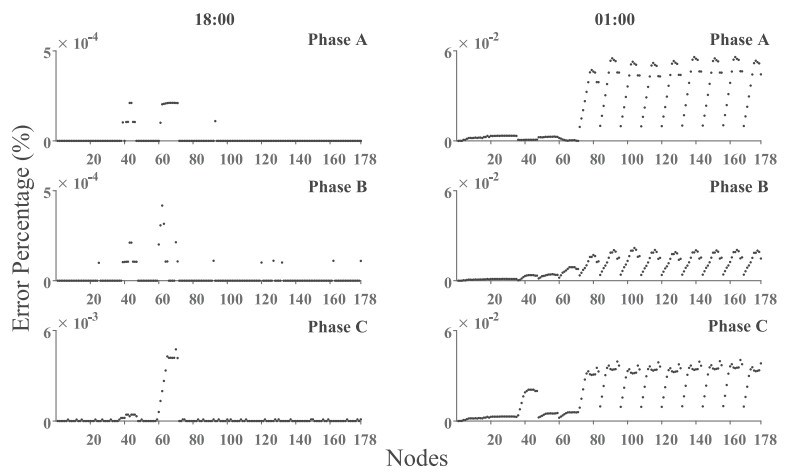

Fig. 5. Voltage magnitude error percentage between the MILP model solution in Case IV and the conventional power flow for time intervals 18:00 and 01:00.

Fig. 5. It is worth noting that this error percentage does not exceed $0.005 \%$ at $18: 00$ hours and $0.06 \%$ at $01: 00$ hours. Hence, these results validate the presented MILP formulation as an accurate approximation of the EDN steady-state operation.

\section{CONCLUSION}

A new methodology based on a mixed-integer linear programming model for the electric vehicle charging coordination (EVCC) problem considering Volt VAr control (VVC), energy storage device (ESD) operation, and dispatchable distributed generation (DG) units in a three-phase unbalanced electrical distribution network (EDN), taking into account a voltage dependent load model, was presented. The proposed formulation was tested on an EDN with 34 medium-voltage nodes and 144 low-voltage nodes. The results showed that the optimized operation of the DG, ESD, VVC devices, and EV recharge represented an overall energy cost reduction and guaranteed the avoidance of technical limit violations.

The dynamic coordination was proved to be efficient, because it defines the step to be implemented and gives a broad view of the EDN state throughout the whole time period. Moreover, this methodology handles the randomness in the electric vehicle (EV) arrival and departure times, initial state of charge, battery sizes, and forecast errors.

The presented results highlight the importance of the proposed methodology, which encompasses the optimal control of the aforementioned devices. Besides, they foreground how the ESDs help in the avoidance of energy curtailment in EV recharging and how the VVC scheme becomes more suitable in order to avoid voltage limit violations in the EDN.

\section{REFERENCES}

[1] M. Biserica, Y. Besanger, R. Caire, O. Chilard, and P. Deschamps, "Neural networks to improve distribution state estimation Volt-VAr control performances," IEEE Trans. Smart Grid, vol. 3, no. 3, pp. 1137-1144, Sep. 2012.

[2] A. Padilha-Feltrin, D. Quijano, and J. R. Mantovani, "Volt-VAr multiobjective optimization to peak-load relief and energy efficiency in distribution networks," IEEE Trans. Power Del., vol. 30, no. 2, pp. 618-626, Apr. 2015.

[3] H. Ahmadi, J. R. Marti, and H. W. Dommel, "A framework for Volt-VAr optimization in distribution systems," IEEE Trans. Smart Grid, vol. 6, no. 3, pp. 1473-1483, May 2015.
[4] K. P. Schneider and T. F. Weaver, "A method for evaluating Volt-VAr optimization field demonstrations," IEEE Trans. Smart Grid, vol. 5, no. 4, pp. 1696-1703, Jul. 2014.

[5] J. Grainger and S. Cinvalar, "Volt/VAr control on distribution systems with lateral branches using shunt capacitors and voltage regulatorsPart I: The overall problem," IEEE Trans. Power App. Syst., vol. PAS104, no. 11, pp. 3278-3283, 1985.

[6] I. Roytelman, B. K. Wee, and R. L. Lugtu, "Volt/VAr control algorithm for modern distribution management system," IEEE Trans. Power Syst., vol. 10, no. 3, pp. 1454-1460, Aug. 1995.

[7] M. E Baran and Ming-Yung Hsu, "Volt/VAr control at distribution substations," IEEE Trans. Power Syst., vol. 14, no. 1, pp. 312-318, Aug. 1995.

[8] V. Borozan, M. E. Baran, and D. Novosel, "Integrated volt/VAr control in distribution systems," in IEEE Proc. Power Eng. Soc. Winter Meeting, Columbus, OH, Jan. 2001, vol. 3, pp. 1485-1490.

[9] B. R. Schainker, "Executive overview: Energy storage options for a sustainable energy future," in Proc. Power Eng. Soc. General Meeting, Jun. 2004, vol. 2, pp. 2309-2314.

[10] L. H. Macedo, J. F. Franco, M. J. Rider, and R. Romero, "Optimal operation of distribution networks considering energy storage devices," IEEE Trans. Smart Grid, vol. 6, no. 6, pp. 2825-2836, Nov. 2015.

[11] I. Atzeni, L. G. Ordonez, G. Scutari, D. P. Palomar, and J. R. Fonollosa "Demand-side management via distributed energy generation and storage optimization," IEEE Trans. Smart Grid, vol. 4, no. 2, pp. 866-876, Jun. 2013.

[12] S. Lin, Z. He, T. Zang, and Q. Qian, "Impact of plug-in hybrid electric vehicles on distribution systems," in Proc. Int. Conf. Power Syst. Technol., 2010, pp. 1-5.

[13] He Yifeng, B. Venkatesh, and Ling Guan, "Optimal scheduling for charging and discharging of electric vehicles," IEEE Trans. Smart Grid, vol. 3, no. 3, pp. 1095-1105, Sep. 2012.

[14] R. Das, K. Thirugnanam, P. Kumar, R. Lavudiya, and M. Singh, "Mathematical modeling for economic evaluation of electric vehicle to smart grid interaction," IEEE Trans. Smart Grid, vol. 5, no. 2, pp. 712-721, Mar. 2014.

[15] A. O'Connell, D. Flynn, and A. Keane, "Rolling multi-period optimization to control electric vehicle charging in distribution networks," IEEE Trans. Power Syst., vol. 29, no. 1, pp. 340-348, Jan. 2014.

[16] S. Deilami, A. S. Masoum, P. S. Moses, and M. A. S. Masoum, "Realtime coordination of plug-in electric vehicle charging in smart grids to minimize power losses and improve voltage profile," IEEE Trans. Smart Grid, vol. 2, no. 3, pp. 456-467, Sep. 2011.

[17] S. Rahimi, M. Marinelli, and F. Silvestro, "Evaluation of requirements for Volt/VAr control and optimization function in distribution management systems," IEEE Int. Energy Conf. Exhibition, Florence, 2012 pp. 331-336.

[18] V. Dabic, S. Cheong, J. Peralta, and D. Acebedo, "BC hydro's experience on voltage VAr optimization in distribution system," IEEE PES Conf. Expo. Trans. Dist., Apr. 2010, pp. 1-7.

[19] L. M. Korunovic, S. Sterpu, S. Djokic, K. Yamashita, S. M. Villanueva, and J. V. Milanovic, "Processing of load parameters based on existing load models," Proc. 3rd IEEE Power Energy Soc. Innovative Smart Grid Technol. Eur., 2012, pp. 1-6.

[20] IEEE task force on load representation for dynamic performance, "Bibliography on load models for power flow and dynamic performance simulation," IEEE Trans. Power Syst., vol. 10, no. 1, pp. 523-538, Feb. 1995.

[21] J. F. Franco, M. J. Rider, and R. Romero, "A mixed-integer linear programming model for the electric vehicle charging coordination problem in unbalanced electrical distribution systems," IEEE Trans. Smart Grid, vol. 6, no. 5, pp. 2200-2210, Sep. 2015.

[22] J. D. Melo, E. M. Carreño, and A. Padilha-Feltrin , "Spatial-temporal simulation to estimate the load demand of battery electric vehicles charging in small residential areas," J. Control, Autom. Elec. Syst., vol. 25 , pp. 470-480, 2014.

[23] K. Qian, C. Zhou, M. Alan, and Y. Yuan, "Modeling of load demand due to EV battery charging in distribution systems," IEEE Trans. Power Sys., vol. 26, no. 2, pp. 802-810, May 2011.

[24] A. Lojowska, D. Kurowicka, G. Papaefthymiou, and L. Van der Sluis, "Stochastic modeling of power demand due to EVs using copula," IEEE Trans. Power Syst., vol. 27, no. 4, pp. 1960-1968, Nov. 2012.

[25] Distribution Test Systems, 33-bus Test System with Secondary Network. Nov. 2016. [Online]. Available: http://www.feis.unesp.br/\#!/ departamentos/engenharia-eletrica/pesquisas-e-projetos/lapsee/

[26] Tesla Motors - Model Specifications. Dec. 2016. [Online]. Available: http://www.teslamotors.com/models/specs 
[27] Nissan-Model Specifications. Dec. 2016. [Online]. Available: http://www.nissa-nusa.com/electric-cars/leaf/

[28] R. Fourer, D. M. Gay, and B. W. Kernighan, AMPL: A Modeling Language for Mathematical Programming, 2nd ed. Pacific Grove, CA, USA: Brooks/Cole-Thomson Learning, 2003.

[29] IBM ILOG CPLEX V12.1 User's Manual for CPLEX, CPLEX Division, ILOG, Inc., Incline Village, NV, USA, 2009.

Carlos Sabillon Antunez received the B.Sc. degree in electrical engineering from the Universidad Nacional Autónoma de Honduras, Tegucigalpa, Honduras, in 2011, M.Sc. degree in electrical engineering from the São Paulo State University (UNESP), Ilha Solteira, Brazil, in 2014, and is currently working toward the Ph.D. degree in electrical engineering at UNESP. His research interests include the development of methodologies for the optimization, planning, and control of electrical power systems.

Ozy D. Melgar-Dominguez received the B.Sc. degree in electrical engineering from the Universidad Nacional Autónoma de Honduras, Tegucigalpa, Honduras, in 2011, the M.Sc. degree in electrical engineering from the São Paulo State University (UNESP), Ilha Solteira, Brazil, in 2015, and is currently working toward the Ph.D. degree in electrical engineering at UNESP. His research interests include the development of methodologies for the optimization, planning, and control of electrical power systems.
John F. Franco (S'11-M'13) received the B.Sc. and M.Sc. degrees in electrical engineering from the Universidad Tecnologica de Pereira, Risaralda, Colombia, in 2004 and 2006, respectively, and the Ph.D. degree in electrical engineering from the São Paulo State University (UNESP), Ilha Solteira, Brazil, in 2012.

$\mathrm{He}$ is currently a Professor in the Electrical Engineering Faculty, UNESP, Rosana, Brazil. His research interests include the development of methodologies for the optimization, planning, and control of electrical power systems.

Marina Lavorato (S'07-M'11) received the B.Sc and M.Sc degrees in 2002 and 2004, respectively, from the Federal University of Juiz de Fora, Brazil, and the Ph.D. degree in 2010 from the University of Campinas (UNICAMP), Campinas, Brazil, all in electrical engineering.

She is a Professor in the Electrical Engineering Faculty, Pontifical Catholic University of Campinas, PUC-CAMPINAS, Campinas, Brazil. Her research interests include the development of methodologies for the optimization, planning, and control of electrical power systems.

Marcos J. Rider (S'97-M'06-SM'16) received the B.Sc.(Hons.) and P.E. degrees from the National University of Engineering, Lima, Peru, in 1999 and 2000, respectively, the M.Sc. degree from the Federal University of Maranhão, Maranhão, Brazil, in 2002; and the Ph.D. degree from the University of Campinas (UNICAMP), Campinas, Brazil, in 2006, all in electrical engineering.

$\mathrm{He}$ is currently a Professor in the Department of Systems and Energy, UNICAMP. His research interests include the development of methodologies for the optimization, planning, and control of electrical power systems, and applications of artificial intelligence in power systems. 